\title{
BASIC NETS IN THE PROJECTIVE PLANE
}

\author{
S. Yu. Orevkov
}

\begin{abstract}
The notion of basic net (called also basic polyhedron) on $S^{2}$ plays a central role in Conway's approach to enumeration of knots and links in $S^{3}$. Drobotukhina applied this approach for links in $\mathbb{R}^{3}$ using basic nets on $\mathbb{R P}^{2}$. By a result of Nakamoto, all basic nets on $S^{2}$ can be obtained from a very explicit family of minimal basic nets (the nets $(2 \times n)^{*}, n \geq 3$, in Conway's notation) by two local transformations. We prove a similar result for basic nets in $\mathbb{R} \mathbb{P}^{2}$.

We prove also that a graph on $\mathbb{R P}^{2}$ is uniquely determined by its pull-back on $S^{2}$ (the proof is based on Lefschetz fix point theorem).
\end{abstract}

\section{Introduction AND STATEMENT OF MAIN RESUlts}

In this paper, a surface is a smooth compact 2-manifold without boundary. A net on a surface $F$ is the image of a generic immersion of several circles.

A net $\Gamma$ is called irreducible if for any embedded circle transversally intersecting $\Gamma$ at most at two points and dividing $F$ into two components $F_{1}$ and $F_{2}$, the following condition holds: one of $F_{1}, F_{2}$ is a disk whose intersection with $\Gamma$ is either a simple arc or empty.

A net $\Gamma$ on $F$ is called basic if it is irreducible and none of the components of $F \backslash \Gamma$ is a digon whose corners are at two distinct vertices.

Basic nets on $S^{2}$ (called in [4] basic polyhedra) were introduced by Conway [4] as a tool for classification of links in $S^{3}$. Drobotukhina [5] applied Conway's approach for links in $\mathbb{R P}^{3}$ using basic nets on $\mathbb{R P}^{2}$

Basic nets on $S^{2}$ (resp. on $\mathbb{R P}^{2}$ ) with $\leq 11$ (resp. with $\leq 8$ ) crossings are shown in Figure 1 (resp. in Figure 2). In all pictures, we represent $\mathbb{R P}^{2}$ as a disk whose opposite boundary points are supposed to be identified. The nets $g_{i}^{n}$ in Figure 2 are denoted as in [5] for $n \leq 6$ (except that $g_{3}^{6}$ is missing in [5]; note that the corresponding alternative link in $\mathbb{R P}^{3}$ also is missing in [5]). For $n \geq 7$ we number them in the order they are produced by plantri program [3] (see §6).

An algorithm to generate all basic nets on $S^{2}$ with a given number of crossings is obtained in [6], improved in [1], and implemented in [3]. The main purpose of the present paper is to extend these results to $\mathbb{R P}^{2}$.

We prove also Theorem 9 which could be of independent interest. It states that any cellular graph on $\mathbb{R P}^{2}$ (i. e. a graph whose complement is a union of open disks) is uniquely determined by its covering on $S^{2}$. The proof is based on the Lefschetz fixed point theorem.

Given a basic net $\Gamma$ of a surface $F$, one can obtain another net using the following transformations:

(1) Face splitting. Suppose that one of the faces of $\Gamma$ is an $n$-gon $f, n \geq 4$. Let $\gamma$ be a simple arc inside $f$ which connects two non-consecutive sides of 


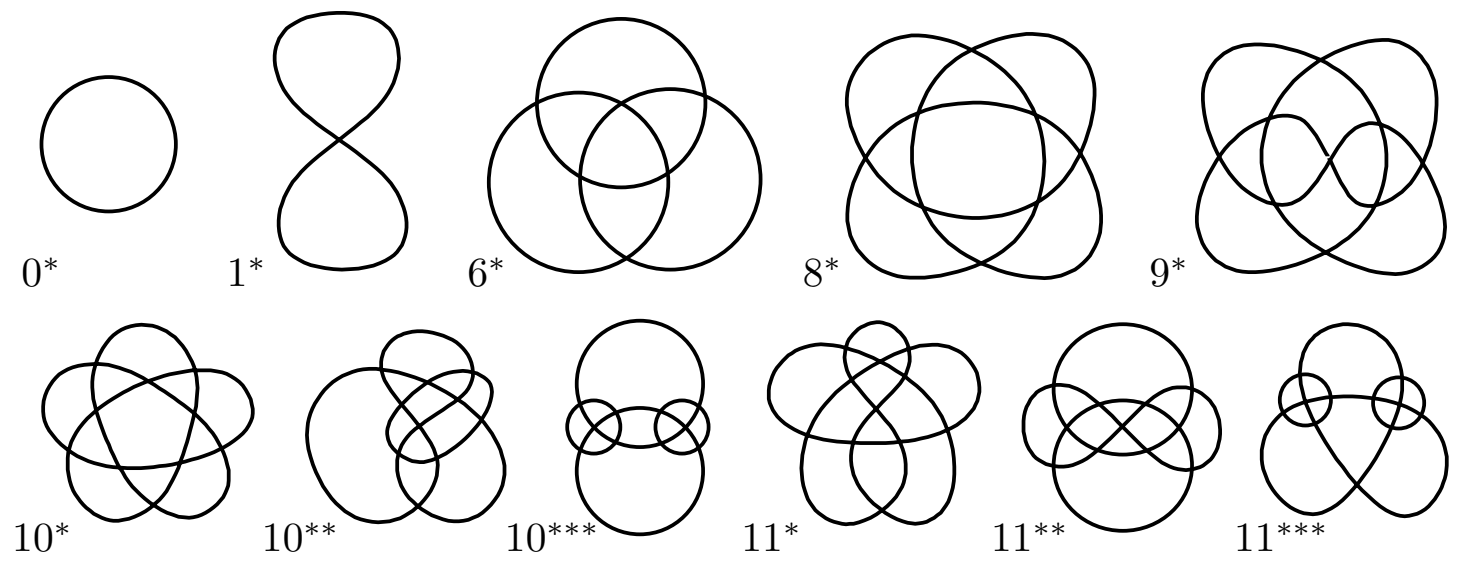

Figure 1. Basic nets on $S^{2}$ with $\leq 11$ nodes
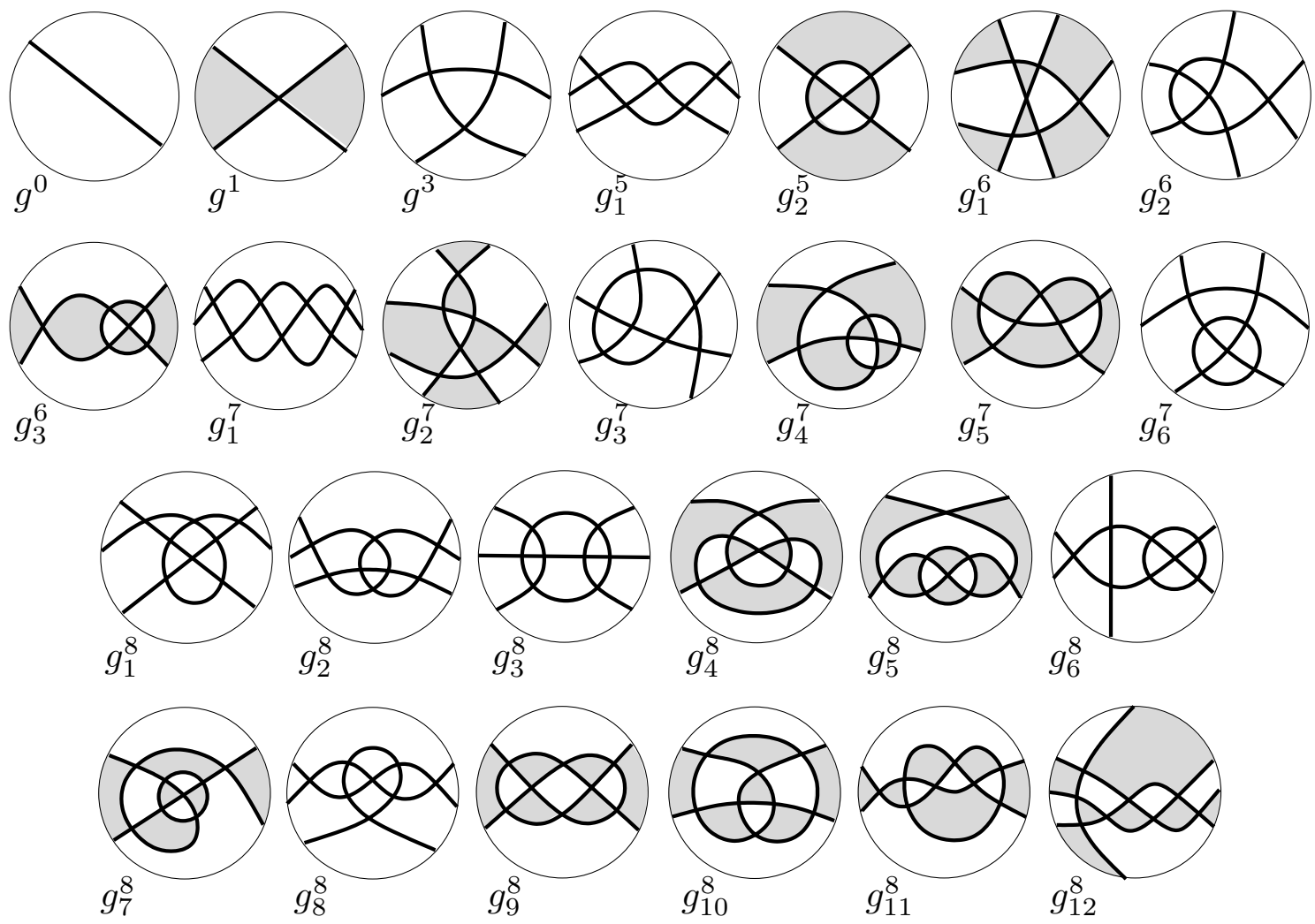

FiguRE 2. Basic nets on $\mathbb{R P}^{2}$ with $\leq 8$ nodes

$f$ represented by two distinct edges of $\Gamma$ (see $\S 2.2$ for a definition of faces, sides and edges). Then a neighbourhood of $\gamma$ is replaced as in Figure 3.1.

(2) Vertex surrounding. A neighbourhood of a vertex of $\Gamma$ is replaced as in Figure 3.2

We say that a face splitting is special if an $n$-gon splits into an $(n-1)$-gon and a triangle.

The nets $6^{*}, 8^{*}$, and $10^{*}$ (Figure 1 ) are the first three members of a series denoted in [4] by $(2 \times n)^{*}, n \geq 3$. The net $(2 \times n)^{*}$ (up to homeomorphism of $S^{2}$ ) is the union of a regular $n$-gon with its inscribed and circumscribed circles. In [6], [1], the dual graph of $(2 \times n)^{*}$ is called pseudo-double wheel and is denoted by $W_{n}$.

A result of Nakamoto [6; Theorem 1] improved in [1; Theorem 2] can be refor- 


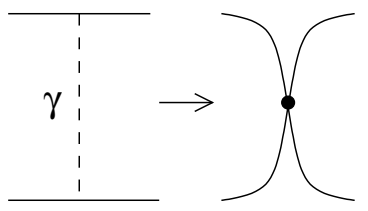

FigurE 3.1. Face splitting

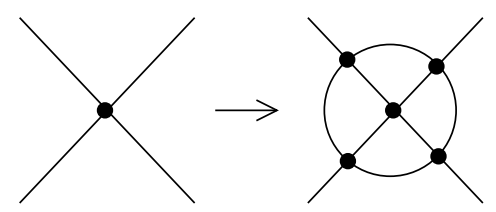

FiguRE 3.2. Vertex surrounding

mulated as follows.

Theorem 1. All basic nets on $S^{2}$ except $0^{*}$ and $1^{*}$ can be obtained from $(2 \times n)^{*}$, $n \geq 3$, by successive special face splittings and vertex surroundings.

In this paper, we generalize Theorem 1 for basic nets in $\mathbb{R P}^{2}$. For an odd $n \geq 3$, let $\overline{(2 \times n)^{*}}$ be the net in $\mathbb{R P}^{2}$ whose double covering is the net $(2 \times n)^{*}$ on $S^{2}$. Due to Theorem 9 in $\S 4, \overline{(2 \times n)^{*}}$ is uniquely determined by this condition. It can be described also as follows. Let $P$ be a regular $n$-gon inscribed in a circle $S$ which bounds a disk $D$. Then, up to homeomorphism, $\overline{(2 \times n)^{*}}$ is the image of $P \cup S$ on the projective plane obtained from $D$ by identifying the opposite boundary points. For $n=3,5,7$, these are the nets $g^{3}, g_{1}^{5}, g_{1}^{7}$ in Figure 2. The dual graph of the net $\overline{(2 \times n)^{*}}$ is called in [6] Möbius wheel and is denoted by $\widetilde{W}_{n}$ (see Figure 4).
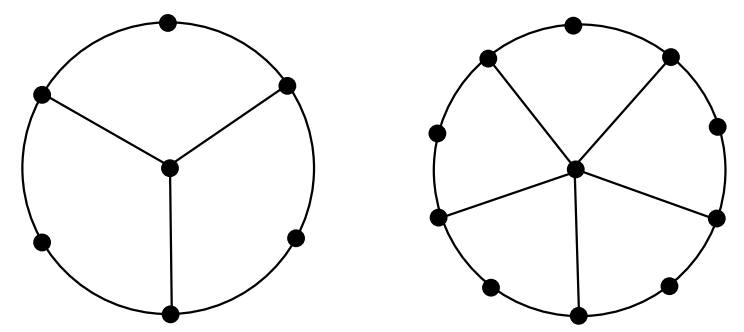

Figure 4. Möbius wheels $\widetilde{W}_{3}$ and $\widetilde{W}_{5}$ in $\mathbb{R P}^{2}$

We say that a net in $\mathbb{R P}^{2}$ is homologically trivial (resp. homologically non-trivial) if it represents zero (resp. non-zero) homology class in $H_{1}\left(\mathbb{R P}^{2} ; \mathbb{Z}_{2}\right)$; in this case the dual graph is bipartite (resp. non-bipartite). In Figure 2 we use the chess-board coloring for the homologically trivial nets. It is easy to see that the face splittings and vertex surroundings do not change the homology class.

Theorem 2. (a). All homologically trivial basic nets on $\mathbb{R P}^{2}$ can be obtained from $g^{1}$ (see Figure 2) by successive special face splittings and vertex surroundings.

(b). All homologically non-trivial basic nets on $\mathbb{R P}^{2}$ except $g^{0}$ can be obtained from $\overline{(2 \times n)^{*}}$ with odd $n \geq 3$ by successive special face splittings and vertex surroundings.

We prove this theorem in $\S 5$.

In Figure 5, we show all the possible special face splittings and vertex surroundings on the basic nets in $\mathbb{R P}^{2}$ with $\leq 8$ crossings. The number of different special face splittings which produce the same result is indicated in parentheses near each arrow. Thus, the list in Figure 2 is exhaustive by Theorem 2 .

Theorem 1 provides an algorithm to generate all basic nets in $S^{2}$. This algorithm is efficiently implemented in the program plantri $[2,3]$. Theorem 2 provides a similar algorithm for $\mathbb{R P}^{2}$ but it is not implemented yet. Instead, we used plantri 


$$
g^{0}
$$
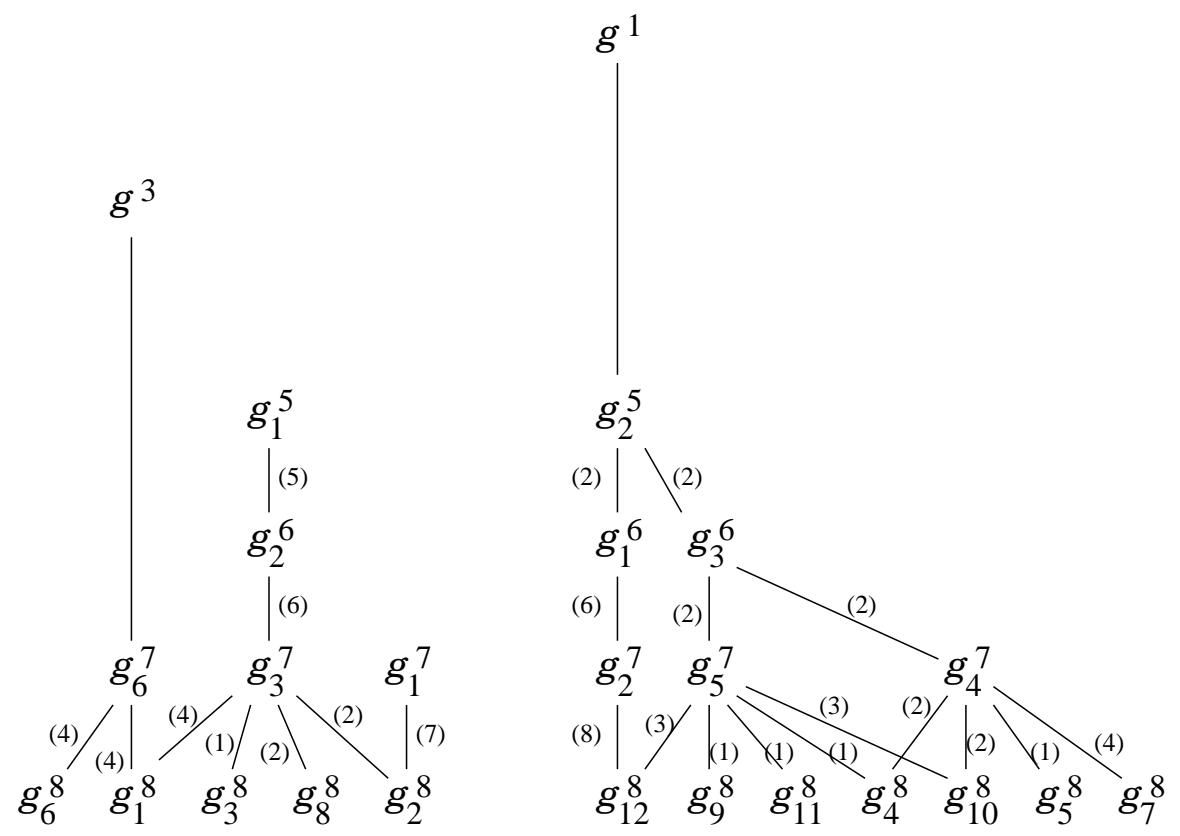

FiguRE 5. Generating basic nets on $\mathbb{R P}^{2}$ with $\leq 8$ crossings

with a simple additional filter [7] to generate all basic nets on $\mathbb{R P}^{2}$ up to 18 crossings, see $\S 6$ for more details.

Acknowledgment. I am grateful to the referee for indicating some mistakes in the first version of this paper.

\section{Definitions}

2.1. Graphs. We shall use the following terminology. A graph $G$ is a triple $(V, E, \partial)$ where $V=V(G)$ and $E=E(G)$ are two sets whose elements are called vertices and edges respectively, and $\partial$ is a mapping from $E(G)$ to the set of unordered pairs of vertices. If $\partial(e)=\{a, b\}$, then $a$ and $b$ are called the ends of $e$. A graph $G$ is called finite if $V(G)$ and $E(G)$ are finite. In this paper we always assume that all graphs are finite and have no isolated vertices.

An edge $e$ is called a loop if $\partial e=\{v, v\}$ for some vertex $v$. Two edges $e$ and $e^{\prime}$ are called parallel if $\partial e=\partial e^{\prime}=\{a, b\}, a \neq b$. A graph is called loop-free if it has no loops. A graph is simple if it is loop-free and has no parallel edges. A simple graph can be defined as a pair $(V, E)$ where $E$ is a set of unordered pairs of distinct vertices.

The number of edges incident to a vertex $v$ (loops counted twice) is called the degree of $v$. We say that a graph is of minimum degree $k$ if the degree of each vertex is at least $k$. A graph is called $k$-regular if the degree of every vertex is $k$.

To each graph we associate a CW-complex of dimension 1 in the standard way. Usually we shall not distinguish between a graph and the corresponding CWcomplex. However, when speaking of graphs, removal of a vertex $v$ always means removal of $v$ together with all the incident edges. So the result is still a graph with one vertex less (not the non-compact space obtained by deleting a vertex from the corresponding CW-complex). 
A graph is called $k$-connected (resp. $k$-edge-connected) if the removal of less than $k$ vertices (resp. edges) cannot disconnect the graph.

2.2. Graphs on surfaces. In this paper, a surface is a smooth compact 2manifold without boundary.

Let $G$ be a graph embedded in a surface $F$. The connected components of $G \backslash F$ are called regions. The pair $(F, G)$ (or just $G$ when it is clear which surface $F$ is considered) is called cellular if each region is homeomorphic to an open disk. In this case the regions are called the faces of $G$. A pair $(F, G)$ is cellular if and only if $F$ admits a structure of CW-complex such that $G$ is the 1-skeleton and $V(G)$ is the 0 -skeleton. It is easy to see that any cellular embedded graph is connected and any connected graph in $S^{2}$ is cellular.

To avoid any ambiguity between an edge (resp. vertex) and its occurrence in the boundary of a given region $r$, we call the latter side (resp. corner) of $r$. In other words, a side (resp. corner) of $r$ is an edge (resp. vertex) adjacent to $r$ which is considered together with a small portion of $r$ near it. The number of sides of a face $f$ is called the degree of $f$. A face of degree $n$ is called also an $n$-gon (union, digon, triangle, quadrangle, pentagon, etc. for $n=1,2,3,4,5, \ldots)$.

A cellular graph is called 2-cell-embedded if all sides and corners of any face are represented by pairwise distinct edges and vertices.

A graph $G$ on a surface $F$ is called simply embedded if it is loop-free and for any two parallel edges $\alpha$ and $\beta$, the circle $\alpha \cup \beta$ does not bound a disk in $F$. In particular, a graph in $S^{2}$ is simply embedded if and only if it is simple.

If $(F, G)$ is cellular, we define the dual graph of $G$ and denote it by $\check{G}$. It has exactly one vertex in each face of $G$ and there is a bijection between the edges of $G$ and those of $\breve{G}$ such that each edge of $\check{G}$ transversally crosses the corresponding edge of $G$ at a single point. According to the previous definition, the degree of a face of $G$ is equal to the degree of the corresponding vertex of $\check{G}$ and vice versa.

A graph $G$ embedded in a surface $F$ is called a quadrangulation of $F$ if all its regions are quadrangles. Note that we do not claim in this definition that $G$ is simple or 2-cell-embedded (as it is demanded in [6] and [1]). For example, if $p$ is a point on the circle $S^{1}$, then $\left(S^{1} \times\{p\}\right) \cup\left(\{p\} \times S^{1}\right)$ is a quadrangulation of the torus $T=S^{1} \times S^{1}$ which has one vertex and two loops. A 3-path (i. e. the graph $\bullet-\bullet-\bullet)$ on a 2 -sphere or a non-contractible 2 -cycle on $\mathbb{R P}^{2}$ are also examples of quadrangulations.

2.3. Basic nets on surfaces. A net on a surface $F$ is the image of a generic immersion of several circles. In particular, a connected net is either a circle or it can be represented by a connected 4-regular embedded graph.

Convention 3. If $\Gamma$ is a connected net which is not an embedded circle, then we consider $\Gamma$ as a 4-regular graph (i. e., all vertices of $\Gamma$ are crossing points).

A net $\Gamma$ is called irreducible if for any embedded circle transversally intersecting $\Gamma$ at most at two points and dividing $F$ into two components $F_{1}$ and $F_{2}$, the following condition holds: one of $F_{1}, F_{2}$ is a disk whose intersection with $\Gamma$ is either a simple arc or empty.

A net $\Gamma$ on $F$ is called basic if it is irreducible and none of the components of $F \backslash \Gamma$ is a digon whose corners are at two distinct vertices.

It is easy to check that in the case when $F$ is $S^{2}$ or $\mathbb{R P}^{2}$, our definition of a basic net is equivalent to the definitions given in [4] and [5] respectively (but our 
definition of an irreducible net differs from that in [5]). Following Conway [4], basic nets on $S^{2}$ are usually called basic polyhedra.

\section{BASIC PROPERTIES OF BASIC NETS}

\subsection{Generalities.}

Proposition 4. Let $\Gamma$ be a basic net on a surface $F$. Then:

(a). Any region of $\Gamma$ is planar, $i$. e., homeomorphic to a subset of $\mathbb{R}^{2}$.

(b). If $F$ is a sphere or $\mathbb{R P}^{2}$, then $\Gamma$ is cellular, in particular, $\Gamma$ is connected.

(c). If $F=\mathbb{R P}^{2}$ and $\Gamma$ is an embedded circle, then $\Gamma$ is a non-contractible curve (a pseudoline).

Proof. (a). Let $r$ be a region of $(F, \Gamma)$. It is an open surface of finite type, thus $r$ is a connected sum of a planar surface and a compact surface without boundary. Thus means that there is an embedded circle $\gamma$ which cuts $r$ into two parts $r_{0}$ and $r_{1}$ such that $r_{0}$ is planar and $\partial r_{1}=\gamma$. Since $(F, \Gamma)$ is irreducible, $r_{1}$ is a disk, hence $r$ is planar.

(b). Let $r$ be a region of $(F, \Gamma)$. By (a), $r$ is planar. Suppose that $r$ has more than one boundary component. Then $r$ can be cut by an embedded circle $\gamma$ into two parts such that each part is adjacent to $\Gamma$. Since $\gamma$ divides $r$, the normal bundle of $\gamma$ is trivial, hence $\gamma$ divides $F$ which contradicts the irreducibility of $(F, \Gamma)$.

(c). Follows from (b).

Proposition 5. Let $F$ be either a sphere or a projective plane. Let $\Gamma$ be a basic net on $F$. Suppose that $\Gamma$ is a 4-regular graph which is not loop-free. Then it has one vertex and two edges (the edges are loops). Moreover, if $F$ is a sphere, then $\Gamma$ is a "figure-eight" curve $\left(1^{*}\right.$ in Figure 1$)$; if $F=\mathbb{R P}^{2}$, then $\Gamma$ is a union of two pseudolines ( $g^{1}$ in Figure 2$)$.

Proof. Suppose that $\Gamma$ has a loop $\alpha$ adjacent to a vertex $v$. Let $N$ be a tubular neighbourhood of $\alpha$ in $F$. It is either an annulus or a Möbius band.

If $N$ is a Möbius band, then $\partial N$ is an embedded circle intersecting $\Gamma$ at two points. Since $\Gamma$ is irreducible, $\partial N$ bounds a disk whose intersection with $\Gamma$ is a simple arc. Then the edge of $\Gamma$ containing this arc is another loop $\beta$ adjacent to $v$. and the result follows (if $\beta$ were not a pseudoline, then $\Gamma$ would be reducible).

Now suppose that $N$ is an annulus. Since $F$ is a sphere or a projective plane, each of the two components of $\partial N$ divides $F$ and intersects $\Gamma$ at most at two points. Hence, the irreducibility of $\Gamma$ implies that $F \backslash N$ is a union of two disjoint disks and $\Gamma \backslash N$ is a simple arc on one of them.

Lemma 6. Let $\Gamma$ be a net in a surface $F$ and let $\alpha$ be a simple closed curve transversal to $\Gamma$ and null-homologous in $F$. Then $\alpha$ cuts $\Gamma$ at an even number of points.

3.2. Basic nets in $S^{2}$. The following fact is well-known but we give a precise statement and a proof for the sake of completeness.

Proposition 7. Let $\Gamma$ be a connected graph on $S^{2}$ and $G$ its dual. The following conditions are equivalent:

(1) $\Gamma$ is a basic net which is neither a circle nor a figure-eight curve; 
(2) $\Gamma$ is simple, 4-regular, and 4-edge-connected;

(3) $G$ is a simple quadrangulation of minimum degree 3 ;

(4) $G$ is a simple, 2-cell-embedded, 2-connected, and 3-edge-connected quadrangulation of minimum degree 3 .

Proof. Note that $\Gamma$ is 4-regular if and only if $G$ is a quadrangulation. So, we assume from now on that $\Gamma$ is 4-regular and $G$ is a quadrangulation.

$(1) \Longrightarrow(2)$. Suppose that Condition (1) holds.

Simplicity. $\Gamma$ is loop-free by Proposition 5. Let us prove that $\Gamma$ cannot have parallel edges. Suppose that $\alpha$ and $\beta$ are two parallel edges. Then $\alpha \cup \beta$ is a simple closed curve (not necessarily smooth). Let $N$ be a tubular neighbourhood of $\alpha \cup \beta$. It is an annulus and the pair $(N, N \cap \Gamma)$ is as in one of Figures 6.1(a-d). Case (d) is impossible by Lemma 6 . In Cases $(\mathrm{a}-\mathrm{c})$, the irreducibility condition implies that one of the components of $\partial N$ (the interior one in Figure 6.1) bounds a disk $D$ such that $D \cap \Gamma$ is $\varnothing$ or an arc. In Cases (a) and (c) this provides a digon and in Case (b) this contradicts the irreducibility (see Figure 6.2). So, we proved that the graph $\Gamma$ is simple.

(a)

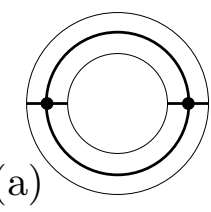

(b)

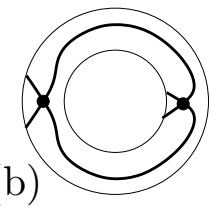

(c)

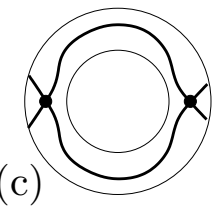

(d)

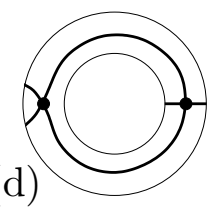

FigURE 6.2.

Figure 6.1.

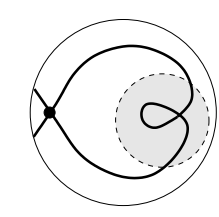

4-edge-connectivity. Let $e_{1}, \ldots, e_{k}$ be a minimal set of edges which disconnects $\Gamma$. Then $\Gamma \backslash \bigcup_{i} e_{i}$ has two connected components $\Gamma_{1}$ and $\Gamma_{2}$, and each edge $e_{i}$ relates them. Hence there exists an embedded circle $\gamma$ which separates $\Gamma_{1}$ from $\Gamma_{2}$ and transversally crosses every edge $e_{i}$ at one point. Hence $k$ is even by Lemma 6 . Since $\Gamma$ is irreducible, $k$ cannot be 0 or 2 . Thus, $k \geq 4$, i. e., $\Gamma$ is 4 -edge-connected.

$(2) \Longrightarrow(1)$. Indeed, the 4-edge-connectivity easily implies the irreducibility and the simplicity (the absence of parallel edges) implies the absence of digons.

$(2) \Longrightarrow(3)$. Suppose that $\Gamma$ is simple and 4-edge-connected.

A loop of $G$ would cut $\Gamma$ at one point which is impossible by Lemma 6 , hence $G$ is loop-free. Suppose that $\alpha$ and $\beta$ are parallel edges of $G$. Then $\alpha \cup \beta$ is a circle which cuts $\Gamma$ at two points. Since $\Gamma$ is irreducible, these two points are connected by a simple arc of $\Gamma$, hence they belong to the same edge of $\Gamma$ which contradicts the definition of the dual graph. Thus, $G$ is simple.

Let us show that the minimum degree is 3 . Indeed, let $v \in V(G)$. If $\operatorname{deg}(v)=1$, then the edge adjacent to $v$ is dual to a loop of $\Gamma$. If $\operatorname{deg}(v)=2$, then the face dual to $v$ is a digon.

$(3) \Longrightarrow(4)$. Suppose that $G$ is simple of minimum degree 3 . Let us show that it is 2-cell-embedded. Indeed, let $f$ be a face of $G$. Suppose that two sides of $f$ are represented by the same edge $e$. If they are consecutive, i. e., if they have a common corner at $v$, then $\operatorname{deg}(v)=1$. If they are opposite, then each of the two other sides represents a loop. So, we conclude that all sides of $G$ are represented by pairwise distinct edges. Suppose that $f$ has two corners at the same vertex. If they 
(a)

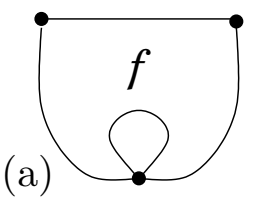

(b)

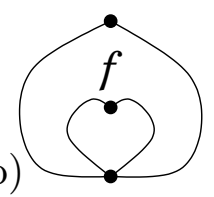

FiguRE 7

are consecutive, then the side between them is a loop (see Figure 7(a)). If they are opposite, then $G$ has parallel edges (see Figure $7(\mathrm{~b})$ ).

$G$ is 2-connected because otherwise it would not be 2-cell-embedded.

Let us show that $G$ is 3-edge-connected. Indeed, let $e_{1}, \ldots, e_{k}$ be a minimal set of edges which disconnects $G$. Then there exists an embedded circle $\gamma$ which transversally crosses every edge $e_{i}$ at one point. We have $k \geq 2$ because $G$ is 2-cellembedded. Suppose that $k=2$. Let $f$ and $f^{\prime}$ be the faces of $G$ crossed by $\gamma$. If $e_{1}$ and $e_{2}$ have a common vertex $v$, then (since $\operatorname{deg} v>2$ ) $v$ represents two corners of one of the faces $f$ or $f^{\prime}$. So, we conclude that $e_{1}$ and $e_{2}$ do not have a common vertex. Then $e_{1}$ and $e_{2}$ are opposite sides of both faces $f$ and $f^{\prime}$. Let $u$ and $v$ be the ends of $e_{1}$ and $e_{2}$ on the same side of $\gamma$. Then $G$ has an edge $u v$ which is a common side of $f$ and $f^{\prime}$. Hence $\operatorname{deg} u=\operatorname{deg} v=2$. Contradiction.

$(4) \Longrightarrow(2)$. Suppose that $G$ is simple, 3 -edge-connected of minimum degree 3. If $\Gamma$ has a loop, then the removal of its dual edge disconnects $G$. If $\Gamma$ has two parallel edges, then the removal of their duals disconnects $G$. Thus, $\Gamma$ is simple. The edge-connectivity of $\Gamma$ is even by Lemma 6 and it cannot be equal to 2 because $G$ has no parallel edges.

\subsection{Basic nets in $\mathbb{R P}^{2}$.}

Theorem 8. Let $\Gamma$ be a cellular graph in $\mathbb{R P}^{2}$ and $G$ its dual. Let $\xi: S^{2} \rightarrow \mathbb{R P}^{2}$ be the universal covering and let $\tilde{\Gamma}=\xi^{-1}(\Gamma)$. Then the following conditions are equivalent:

(1) $\Gamma$ is a basic net which is neither a line nor a union of two lines;

(2) $\Gamma$ is simply embedded, 4-regular, 4-edge-connected, and $G$ is loop-free;

(3) $G$ is a simply embedded quadrangulation of minimum degree 3 ;

(4) $\tilde{\Gamma}$ is a basic net in $S^{2}$ which is not a circle.

Proof. (1) $\Longrightarrow(2)$. Assume that Condition (1) holds. Let us show that $G$ is loopfree. Indeed, if $\alpha$ is a loop of $G$, then it cuts $\Gamma$ at one point. By Lemma 6 , this implies that $\alpha$ is a pseudoline. Let $N$ be a tubular neighbourhood of $\alpha$ and let $D=\mathbb{R P}^{2} \backslash N$. Then $N$ is a Möbius band and $\Gamma \cap N$ is a simple arc, in particular, $\partial N$ is a circle which cuts $\Gamma$ at two points. Then $D \cap \Gamma$ is a also simple arc because $\Gamma$ is irreducible, i. e., $\Gamma$ is a circle which is impossible by Condition (1). Thus, $G$ is loop-free. The rest of the proof is the same as in Proposition 7.

$(2) \Longrightarrow(3)$. The same proof as in Proposition 7 (note that $G$ is already loop-free by Condition (2)).

$(3) \Longrightarrow(4)$. It is immediate to check that if $G$ is a simply embedded quadrangulation of $\mathbb{R P}^{2}$ of minimum degree 3 , then $\xi^{-1}(G)$ is a simple quadrangulation of $S^{2}$ of minimum degree 3. Thus, the result follows from Proposition 7.

$(4) \Longrightarrow(1)$. Assume that $\tilde{\Gamma}$ is a basic net in $S^{2}$ and let us prove that $\Gamma$ is a basic net in $\mathbb{R P}^{2}$. If $D$ is a digon of $\Gamma$, then $\xi^{-1}(D)$ is a digon of $\tilde{\Gamma}$, thus it remains 
to prove that $\Gamma$ is irreducible. Indeed, let $\gamma$ be an embedded circle transversally intersecting $\Gamma$ at $i \leq 2$ points and dividing $\mathbb{R P}^{2}$ into two components $(i=0$ or 2 by Lemma 6). One of the components is an open disk $D$. Let $\xi^{-1}(D)=\tilde{D}_{1} \sqcup \tilde{D}_{2}$ and $\tilde{\gamma}_{1}=\partial \tilde{D}_{1}$. If $i=0$, then $D \cap \Gamma=\varnothing$ because $\tilde{\Gamma}$ is connected. Let $i=2$. Since $\tilde{\Gamma}$ is irreducible, $\tilde{D} \cap \tilde{\Gamma}$ is a simple arc where $\tilde{D}$ is one of the two components of $S^{2} \backslash \tilde{\gamma}_{1}$. If $\tilde{D}=\tilde{D}_{1}$, then $D \cap \Gamma=\xi(\tilde{D} \cap \tilde{\Gamma})$ is a simple arc and we are done. Otherwise $\tilde{\Gamma} \backslash \tilde{D}_{1}$ is a simple arc, hence its subset $\tilde{\Gamma} \cap \tilde{D}_{2}$ is a priori a disjoint union of simple arcs, but the total number of their boundary points is 2 , hence it is a simple arc.

\section{Uniqueness of A PLANAR PROJECTIVE QUOTIENT OF A PLANAR GRAPH.}

Theorem 9. Let $G_{1}$ and $G_{2}$ be embedded graphs in $\mathbb{R P}^{2}$ without vertices of degree 2. Let $\xi_{1}$ and $\xi_{2}$ be two unramified coverings $S^{2} \rightarrow \mathbb{R P}^{2}$ and let $\sigma_{j}: S^{2} \rightarrow S^{2}$, $j=1,2$, be the corresponding deck transformations, $i$. e., for any $x \in S^{2}, \sigma_{j}(x)=y$ where $y \neq x$ and $\xi_{j}(x)=\xi_{j}(y)$. Suppose that $\xi_{1}^{-1}\left(G_{1}\right)=\xi_{2}^{-1}\left(G_{2}\right)$ and that it is a connected graph (we denote it by $G$ ). Then $\left.\sigma_{1}\right|_{G}$ and $\left.\sigma_{2}\right|_{G}$ are combinatorially equivalent, i. e., $\left.\sigma_{1}\right|_{V(G)}=\left.\sigma_{2}\right|_{V(G)}$ and for any $e \in E(G)$ we have $\sigma_{1}(e)=\sigma_{2}(e)$.

Proof. Without loss of generality we may assume that $S^{2}$ is glued out of regular polygons ( $G$ being represented by their sides) and the mappings $\sigma_{j}$ are linear on each of them. Then $\sigma_{1}$ and $\sigma_{2}$ are combinatorially equivalent if and only if $\sigma_{1}=\sigma_{2}$.

We set $\tau=\sigma_{1} \circ \sigma_{2}$. Since $\sigma_{1}^{2}=\sigma_{2}^{2}=\operatorname{id}_{S^{2}}$, it is enough to prove that $\tau=\operatorname{id}_{S^{2}}$. Note that $\tau$ is an orientation preserving homeomorphism $S^{2} \rightarrow S^{2}$. We suppose that $\tau \neq$ id and we shall obtain a contradiction in several steps.

Step 1. There do not exist $v \in V(G)$ and an edge e adjacent to $v$ such that $\tau(v)=v$ and $\tau(e)=e$. Indeed, $\tau$ is the identity map on the faces adjacent to $v$. The same is true for faces adjacent to them etc. Since the graph $G$ is connected, we exhaust all its vertices and edges by this process.

Step 2. $\tau$ has exactly two fix points. Indeed, let $L(\tau)$ be the Lefschetz number of $\tau$, i. e., $L(\tau)=\sum_{q}(-1)^{q} \operatorname{trace}\left(\tau_{*}: H_{q}\left(S^{2}\right) \rightarrow H_{q}\left(S^{2}\right)\right)$. Since $\tau$ is an orientation preserving homeomorphism, we have $L(\tau)=2$. It is well-known that $L(\tau)$ is equal to the intersection number of the diagonal of $S^{2} \times S^{2}$ with the graph of $\tau$. We deduce from the result of Step 1 that the number of fix points of $\tau$ is finite. Moreover, at any fix point, $\tau$ is locally conjugated to a rotation, hence the local intersection of the diagonal with the graph at any fix point is equal to +1 .

Step 3. We denote the fix points of $\tau$ by $x$ and $y$. Then $\sigma_{1}(x)=\sigma_{2}(x)=y$ and $\sigma_{1}(y)=\sigma_{2}(y)=x$. Indeed, let $z=\sigma_{2}(x)$. By the definition of $\tau$ we have $\tau(z)=\sigma_{1}\left(\sigma_{2}\left(\sigma_{2}(x)\right)\right)=\sigma_{1}(x)$. Since $x$ is a fix point of $\tau$, we have $\sigma_{1}(x)=$ $\sigma_{1}(\tau(x))=\sigma_{1}\left(\sigma_{1}\left(\sigma_{2}(x)\right)\right)=\sigma_{2}(x)=z$. Thus $\tau(z)=\sigma_{1}(x)=z$, i. e., $z$ is a fix point of $\tau$. Hence $z=y$. The other equalities are obtained similarly.

Step 4. Subdividing if necessary the faces containing $x$ and $y$ we may assume without loss of generality that $x$ and $y$ are vertices of $G$. Let $\gamma$ be a shortest path on $G$ from $x$ to $y$ (a path with the minimum number of edges). Let $x=x_{0}, x_{1}, \ldots, x_{n}=$ $y$ be the successive vertices on $\gamma$. Then $\sigma_{1}(\gamma) \cap \sigma_{2}(\gamma)=\{x, y\}$. Indeed, Suppose that $\sigma_{1}\left(x_{i}\right)=\sigma_{2}\left(x_{j}\right)$. If $i \neq j$, say, $i<j$, then $\gamma$ is not a shortest path from $x$ to $y$ because in this case the path $x=\tau\left(x_{0}\right), \tau\left(x_{1}\right), \ldots, \tau\left(x_{i}\right)=x_{j}, x_{j+1}, \ldots, x_{n}=y$ is yet shorter. Hence $i=j$ and so $x_{i}$ is a fix point of $\tau$, hence $x_{i}=x_{j} \in\{x, y\}$. 
Thus, $\sigma_{2}(\gamma)$ is contained in one of the two disks bounded by the circle $\gamma \cup \sigma_{1}(\gamma)$. This contradicts the fact that each of the circles $\gamma \cup \sigma_{1}(\gamma)$ and $\gamma \cup \sigma_{2}(\gamma)$ divides the sphere into two halves containing the same number of 2 -faces.

\section{Generating Basic nets}

In this section we prove Theorem 2. By the duality (see Theorem 8), it follows easily from Theorem 12 (see below) combined with Theorem 1.

5.1. Face contraction/removal. We recall here some definitions from [6], [1].

Let $G$ be a quadrangulation of a surface $F$ which has more than one face and let $f=a b c d$ be a face of $G$ such that $a \neq c$. In this case we say that the face $f$ is contractible at $\{a, c\}$ and the contraction of $f$ at $\{a, c\}$ consists in the removal of the interior of $f$ and glueing the edges $b a$ with $b c$ and $d a$ with $d c$ (see Figure 11). The inverse operation to a face contraction is called a vertex splitting.
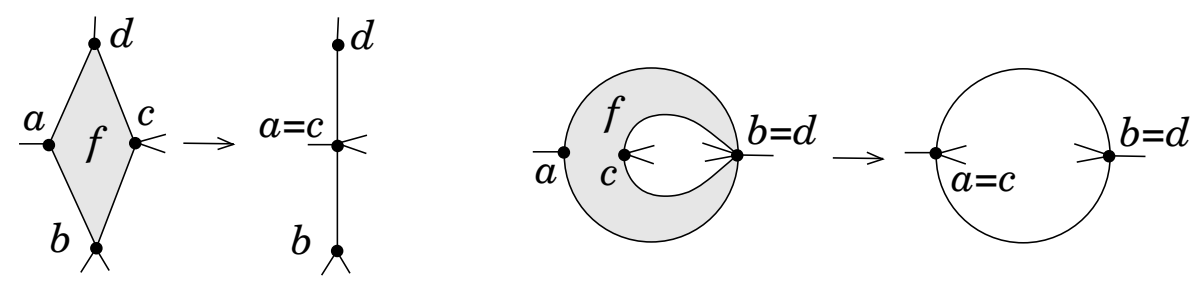

Figure 11. Examples of face contraction

Let $G$ be a quadrangulation of a surface $F$ and let $f=a b c d$ be a face of $G$. We say that $f$ is removable if $a, b, c, d$ are pairwise distinct vertices of degree 3 and, if we denote their outcoming edges (not being the sides of $f$ ) by $a a_{1}, b b_{1}, c c_{1}, d d_{1}$, then $\{a, b, c, d\} \cap\left\{a_{1}, b_{1}, c_{1}, d_{1}\right\}=\varnothing$. In this case, the removal of $f$ consists just in the removal of the vertices $a, b, c, d$ and all the edges incident to them (see Figure 12). The inverse operation to a face removal is called a face addition.
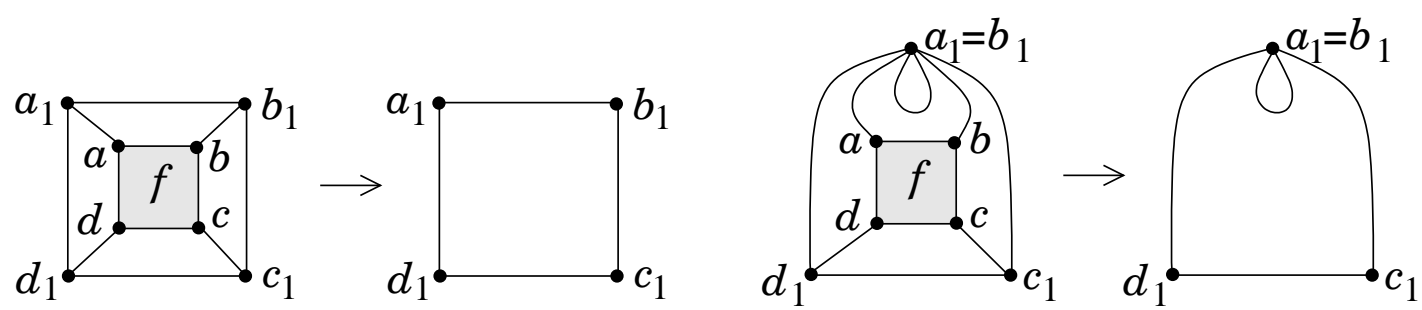

Figure 12. Examples of face removal

It is easy to see that the result of a face contraction/removal is again a quadrangulation of the same surface.

Let $\mathcal{Q}$ (resp. $\overline{\mathcal{Q}}$ ) be the class of simple (resp. simply embedded) quadrangulations of $S^{2}$ (resp. of $\mathbb{R P}^{2}$ ) of minimal degree 3. By Proposition 7 (resp. by Theorem 8), it is the dual of the class of basic nets on $S^{2}$ (resp. on $\mathbb{R P}^{2}$ ) with more than one crossing.

Given $G \in \mathcal{Q}$, we say that a face $a b c d$ of $G$ is $\mathcal{Q}$-removable (resp. $\mathcal{Q}$-contractible at $\{a, c\})$ if it is removable (resp. contractible at $\{a, c\}$ ) and the result of the 
removal (resp. contraction) belongs to $\mathcal{Q}$. We say that a $\mathcal{Q}$-contraction of a face $a b c d$ at $\{a, c\}$ is special if $\operatorname{deg} a=3$ or $\operatorname{deg} c=3$.

If $G \in \mathcal{Q}$ does not have any special $\mathcal{Q}$-contractible or $\mathcal{Q}$-removable face, then we say that $G$ is $\mathcal{Q}$-minimal.

In the same way we define (special) $\overline{\mathcal{Q}}$-contractible/removable faces (of quadrangulations belonging to $\overline{\mathcal{Q}}$ ) and $\overline{\mathcal{Q}}$-minimal quadrangulations. Let

$$
\mathcal{Q}_{\text {min }}=\{G \in \mathcal{Q} \mid G \text { is } \mathcal{Q} \text {-minimal }\}, \quad \overline{\mathcal{Q}}_{\text {min }}=\{G \in \overline{\mathcal{Q}} \mid G \text { is } \overline{\mathcal{Q}} \text {-minimal }\}
$$

It is clear that (special) $\mathcal{Q}$ - or $\overline{\mathcal{Q}}$-vertex-splittings and $\mathcal{Q}$ - or $\overline{\mathcal{Q}}$-face-additions are dual to (special) face splittings and vertex surroundings on basic nets respectively.

5.2. Double covering and minimality. Let $\xi: S^{2} \rightarrow \mathbb{R P}^{2}$ be the double covering and $\sigma: S^{2} \rightarrow S^{2}$ its deck transformation, i. e., $\xi \circ \sigma=\xi$ and $\sigma \neq$ id. For $a \in S^{2}$ or $a \subset S^{2}$, we denote $\sigma(a)$ by $a^{\prime}$ and $\xi(a)$ by $\bar{a}$.

Lemma 10. Let $\bar{G} \in \overline{\mathcal{Q}}$ and $G=\xi^{-1}(\bar{G})$. Suppose that a face $f=$ abcd of $G$ is $\mathcal{Q}$-contractible at $\{a, c\}$. Then:

(a) The vertices $a, a^{\prime}, c, c^{\prime}$ are pairwise distinct.

(b) $\bar{f}$ is not $\overline{\mathcal{Q}}$-contractible at $\{\bar{a}, \bar{c}\}$ if and only if one of the following two conditions holds: (i) $G$ has an edge $a c^{\prime}$ or (ii) $b=d^{\prime}$ and $\operatorname{deg} b=4$.

(c) Assume, moreover, that $f$ is special $\mathcal{Q}$-contractible at $\{a, c\}$. Then $\bar{f}$ is special $\overline{\mathcal{Q}}$-contractible at $\{\bar{a}, \bar{c}\}$ if and only if ac $\notin E(G)$.

Proof. (a). We have $a \neq c$ because $G \in \mathcal{Q}$ by Theorem 8 , hence $G$ is 2-cellembedded by Proposition 7 . We have $a \neq c^{\prime}$ because otherwise we have $f^{\prime}=$ $a^{\prime} b^{\prime} c^{\prime} d^{\prime}=c b^{\prime} a d^{\prime}$ and the result of the contraction of $f$ is not 2-cell-embedded at the face $f^{\prime}$ which contradicts Proposition 7.

(b). By (a) we have $\bar{a} \neq \bar{c}$, i. e., the face $\bar{f}$ is contractible at $\{\bar{a}, \bar{c}\}$. Let $\bar{G}_{1}$ be the result of the contraction.

If (i) holds, then the image of the edge $\bar{a} \bar{c}$ on $\bar{G}_{1}$ would be a loop; if (ii) holds, then $\operatorname{deg}_{\bar{G}_{1}} \bar{b}=2$. In both cases we have $\bar{G}_{1} \notin \overline{\mathcal{Q}}$.

Suppose that none of Conditions (i), (ii) holds. Let us show that $\bar{G}_{1} \in \overline{\mathcal{Q}}$. It is clear that $\bar{G}_{1}$ is a quadrangulation. Since $f$ is $\mathcal{Q}$-contractible, we have $\operatorname{deg} \bar{b}=$ $\operatorname{deg} b>3$ and $\operatorname{deg} \bar{d}=\operatorname{deg} d>3$. Since Condition (ii) does not hold, it follows that $\bar{G}_{1}$ is a quadrangulation of minimum degree 3 . So, it remains to prove that $\bar{G}_{1}$ is simply embedded. Since $\bar{G}$ is loop-free and $a \neq c^{\prime}$, it follows that $\bar{G}_{1}$ is loop-free also. Suppose that there are two parallel edges on $\bar{G}_{1}$ which bound a disk $D^{*}$ on $\mathbb{R P}^{2}$. Let $\pi: \mathbb{R P}^{2} \rightarrow \mathbb{R P}^{2}$ be a continuous mapping which extends the contraction of $\bar{f}$ so that $\left.\pi\right|_{\bar{f}}$ is constant on each segment parallel to the diagonal $\bar{a} \bar{c}$ and $\left.\pi\right|_{\mathbb{R P}^{2} \backslash \bar{f}}$ is a homeomorphism onto its image. Then $\bar{D}=\pi^{-1}\left(D^{*}\right)$ is a disk bounded by two edges of $\bar{G}$ and, maybe, by the diagonal of $\bar{f}$ if $\pi(\bar{a})=\pi(\bar{c}) \in \partial D^{*}$. Thus, $\xi^{-1}(\bar{D})$ is a disjoint union of two disks $D \cup D^{\prime}$ on $S^{2}$ such that $D$ is bounded by two edges of $G$ and, maybe, by the diagonal $a c$ of the face $f$. Thus either $G$ or the result of the contraction of $f$ at $\{a, c\}$ is not simple. Contradiction.

(c). Since $f$ is special $\mathcal{Q}$-contractible at $\{a, c\}$, without loss of generality we may assume that $\operatorname{deg}(a)=3$. By (b), it is enough to show that Condition (ii) does not hold. Suppose that it does hold. Then, by (a), $G$ contains a subgraph depicted in Figure 13.1. Here we denote the third outcoming edge from $a$ by $a x$. It is clear that $x$ should be in the quadrangle $q=a b c^{\prime} d$. It cannot be on the boundary of 
$q$. Indeed, $x \notin\{a, b, d\}$ because $G$ is simple and $x \neq c^{\prime}$ because $G$ is bipartite (see the colors in Figure 13.1). We have $\operatorname{deg}(b)=\operatorname{deg}(d)=4$ and $\operatorname{deg}(a)=3$, hence all outcoming edges from $a, b$, and $d$ are already present in Figure 13.1. Therefore, the path $x a b c^{\prime}$ follows the boundary of the same face (we denote it by $f_{1}$ ). Hence $G$ has an edge $x c^{\prime}$ adjacent to $f_{1}$. Similarly, $x c^{\prime}$ is adjacent to the face $f_{2}=x a d c^{\prime}$. Since $f_{1} \cup f_{2}=q$, we conclude that $\operatorname{deg}(x)=2$. Contradiction.

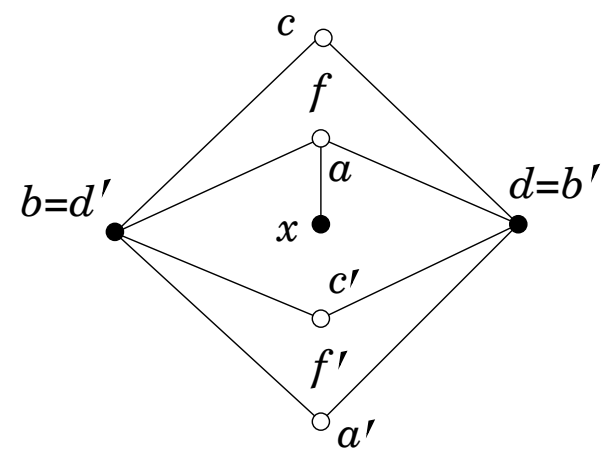

FiguRE 13.1

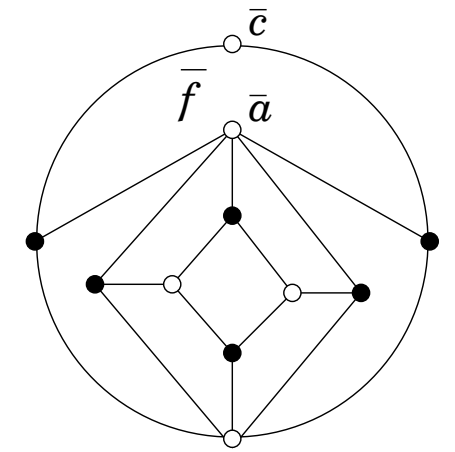

FiguRE $13.2 \check{g}_{5}^{8}$

Remark. Condition (ii) of Lemma 10(b) holds for the quadrangulation $\bar{G}=\check{g}_{5}^{8}$ depicted in Figure 13.2 which is dual to the basic net $g_{5}^{8}$ in Figure 2. In this case $f$ is (non-special) $\mathcal{Q}$-contractible at $\{a, c\}$ but $\bar{f}$ is not $\overline{\mathcal{Q}}$-contractible at $\{\bar{a}, \bar{c}\}$.

Lemma 11. Let $\bar{G} \in \overline{\mathcal{Q}}$ and let $G=\xi^{-1}(\bar{G})$. Suppose that a face $f_{0}$ of $G$ is removable. Let $f_{1}, f_{2}, f_{3}, f_{4}$ be the faces which have a common edge with $f_{0}$. If $f_{i} \neq f_{j}^{\prime}$ for any $i, j \in\{0, \ldots, 4\}$, then $\bar{f}_{0}$ is removable.

Proof. Suppose that $\bar{f}_{0}$ is not removable. Than $\bar{x}=\bar{y}$ for a vertex $x$ of $f_{0}$ and for a vertex $y \neq x$ of one of $f_{0}, \ldots, f_{4}$. Since $x \neq y$ and $\bar{x}=\bar{y}$, it follows that $y^{\prime}=x$. One of the faces adjacent to $y$ is $f_{i}$ for some $i=0, \ldots, 4$. Then $f_{i}^{\prime}$ is adjacent to $y^{\prime}=x$. Since any face adjacent to $x$ is one $f_{0}, \ldots, f_{4}$, it follows that $f_{i}^{\prime}=f_{j}$ for some $j=0, \ldots, 4$.

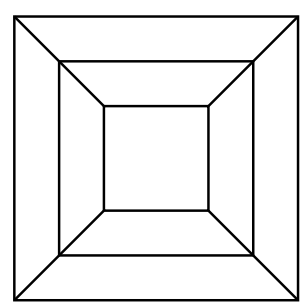

FiguRE 14. The covering of $\check{g}_{2}^{5}$

Theorem 12. Let $\bar{G} \in \overline{\mathcal{Q}}_{\min }$ and $G=\xi^{-1}(\bar{G})$. Then either $G \in \mathcal{Q}_{\min }$ or $G$ is as in Figure 14 (and then $\bar{G}=\check{g}_{2}^{5}$ - the dual of the basic net $g_{2}^{5}$ in Figure 2).

Proof. Suppose that $G \notin \mathcal{Q}_{\min }$. Then it admits either a special $\mathcal{Q}$-face-contraction or a $\mathcal{Q}$-face-removal.

Case 1. $G$ admits a special $\mathcal{Q}$-contraction of a face $f=a b c d$ at $\{a, c\}$. We may assume that $\operatorname{deg}(a)=3$. Since $\bar{G} \in \overline{\mathcal{Q}}_{\text {min }}$, the face $\bar{f}$ is not $\overline{\mathcal{Q}}$-contractible at $\{\bar{a}, \bar{c}\}$. 


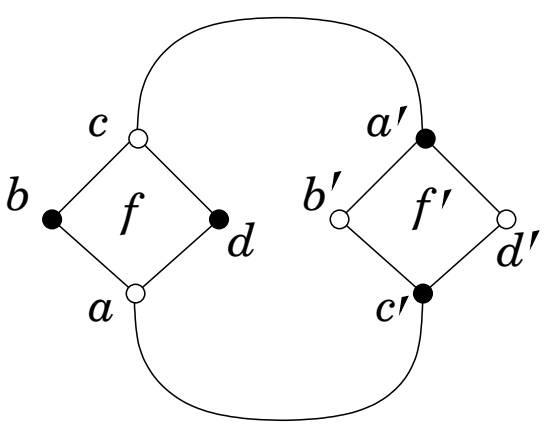

FiguRE 15.1

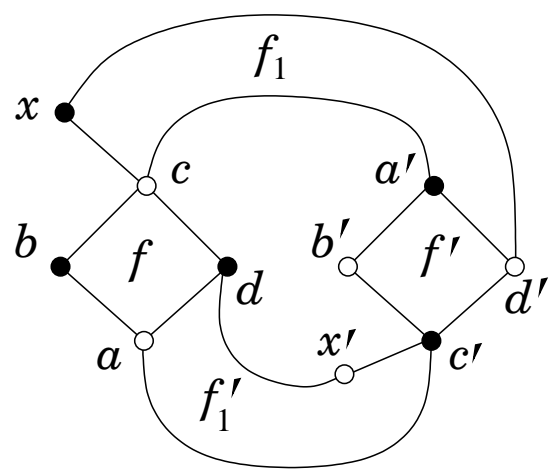

FiguRE 15.2

By Lemma 10(c), this implies that $G$ has edges $a c^{\prime}$ and $c a^{\prime}$ (note that $a \neq c^{\prime}$ by Lemma 10(a)). Then $b^{\prime} \neq d$ because $G$ is bipartite, hence $G$ contains a subgraph $H$ shown in Figure 15.1.

Subcase 1.1. $\operatorname{deg}(c)=3$. In this case the paths $b a c^{\prime} d^{\prime}, d a c^{\prime} b^{\prime}, d c a^{\prime} b^{\prime}$, and $b c a^{\prime} d^{\prime}$ belong to the boundaries of some faces. Hence there are edges $b d^{\prime}$ and $b^{\prime} d$ and when we add them to $H$, we complete the graph $G$. Then $\operatorname{deg}(b)=\operatorname{deg}(d)=3$ which contradicts the condition that $f$ is $\mathcal{Q}$-contractible at $\{a, c\}$.

Subcase 1.2. $\operatorname{deg}(c)>3$. Let $e=c x$ be an edge adjacent to $c$ which is not in $H$ and which is next to $c a^{\prime}$ in the natural cyclic order on the set of edges outcoming from $c$. Since $H$ is symmetric, we may assume that $e$ sits in the hexagon $h=$ $a b c a^{\prime} d^{\prime} c^{\prime}$ (the exterior region in Figure 15.1). The vertex $x$ is not on the boundary of $h$. Indeed, $x \notin\left\{a, d^{\prime}\right\}$ because $G$ is bipartite (see the colors in Figure 15.1), $x \notin\left\{b, a^{\prime}\right\}$ because $G$ is simple, and $x \neq c^{\prime}$ because $\bar{G}$ is loop-free. Thus, $x c a^{\prime} d^{\prime}$ is a path in $G$. Moreover, by the assumption that $\operatorname{deg}(a)=3$, this path belongs to the boundary of some face $f_{1}$ (see Figure 15.2). Then $\bar{f}_{1}$ is $\overline{\mathcal{Q}}$-contractible at $\{\bar{a}, \bar{x}\}$ by Lemma 10. Indeed, we have $\operatorname{deg}(c)>3$ by hypothesis, $\operatorname{deg}\left(d^{\prime}\right)=\operatorname{deg}(d)>3$ because $f$ is $\mathcal{Q}$-contractible at $\{a, c\}$, and there is no edge $x a$ because $\operatorname{deg}(a)=3$ and we have already three outcoming edges from $a$. Moreover, $\bar{f}_{1}$ is special $\overline{\mathcal{Q}}$ contractible at $\{\bar{a}, \bar{x}\}$ because $\operatorname{deg} \bar{a}=\operatorname{deg} a=3$.

Case 2. $G$ has a $\mathcal{Q}$-removable face $f=$ abcd. Let $a_{1}, b_{1}, c_{1}, d_{1}$ be as in the definition of the face removal (see $\S 5.1$ ) and let $G_{1}$ be the result of the removal of the face $f$. Since $G_{1}$ is in $\mathcal{Q}$, it is 2-cell-embedded by Proposition 7 , hence $a_{1}, b_{1}, c_{1}, d_{1}$ are pairwise distinct. Since $f$ is $\mathcal{Q}$-removable, we have

$$
\operatorname{deg} x>3 \quad \text { for } x \in\left\{a_{1}, b_{1}, c_{1}, d_{1}\right\} .
$$

Let us prove that $\bar{f}$ is removable. By Lemma 11 and by symmetry, it suffices to check that $a^{\prime} \notin\left\{a, b, c, d, a_{1}, b_{1}, c_{1}, d_{1}\right\}$. We have $a^{\prime} \notin\left\{a_{1}, b_{1}, c_{1}, d_{1}\right\}$ by (1), $a^{\prime} \neq a$ because $\sigma$ has no fix point, and $a^{\prime} \neq b$ because $\bar{G}$ is loop-free. Suppose that $a^{\prime}=c$. Then $b^{\prime}$ is connected to $c$ by an edge. i. e., $b^{\prime} \in\left\{b, d, c_{1}\right\}$. We have $b^{\prime} \neq b$ (no fix point of $\sigma$ ) and $b \neq c_{1}$ by $(1)$, hence $b^{\prime}=d$. Thus, $\sigma$ maps the edge $a b$ to the edge $c d$. If follows that the face $f^{\prime}$ is incident to $c d$. This is impossible because $f^{\prime} \neq f$ (otherwise $\sigma$ has a fix point) and $f^{\prime} \neq c d d_{1} c_{1}$ by (1). So, we proved that $\bar{f}$ is removable.

Let $\bar{G}_{1}$ be the result of the removal of the face $\bar{f}$. Then $\bar{G}_{1}$ is a simply embedded quadrangulation and $\operatorname{deg}_{\bar{G}_{1}}(x) \geq 3$ for $x \notin\left\{\bar{a}_{1}, \bar{b}_{1}, \bar{c}_{1}, \bar{d}_{1}\right\}$. Since $\bar{G} \in \overline{\mathcal{Q}}_{\text {min }}$, we know that $\bar{G}_{1} \notin \overline{\mathcal{Q}}$. Hence the degree in $\bar{G}_{1}$ of one of $\bar{a}_{1}, \bar{b}_{1}, \bar{c}_{1}, \bar{d}_{1}$, (say, $\bar{a}_{1}$ ) is less 
that 3. Since $\operatorname{deg}_{\bar{G}}\left(\bar{a}_{1}\right)=\operatorname{deg}_{G}\left(a_{1}\right)>3$ (see (1)), this means that $\bar{a}_{1}$ is incident in $\bar{G}$ to at least two edges which are removed in $\bar{G}_{1}$. This may happen only if $a_{1}^{\prime} \in\left\{a_{1}, b_{1}, c_{1}, d_{1}\right\}$. We have $a_{1}^{\prime} \neq a_{1}$ (since $\sigma$ has no fix point) and $a_{1}^{\prime} \notin\left\{b_{1}, d_{1}\right\}$ (since $\bar{G}$ is loop-free), hence $a_{1}^{\prime}=c_{1}$. We have $\operatorname{deg}_{G}\left(a_{1}\right)>3, \operatorname{deg}_{\bar{G}_{1}}\left(\bar{a}_{1}\right)<3$, and $\operatorname{deg}_{\bar{G}_{1}}\left(\bar{a}_{1}\right)=\operatorname{deg}_{G}\left(a_{1}\right)-2$, hence $\operatorname{deg}_{G}\left(a_{1}\right)=4$ and $\operatorname{deg}_{\bar{G}_{1}}\left(\bar{a}_{1}\right)=2$. This means that the only vertices connected to $a_{1}$ are $a, c^{\prime}, b_{1}, d_{1}$. Since $a_{1}^{\prime}=c_{1}$, we have $\operatorname{deg}_{G}\left(c_{1}\right)=4$, hence the vertices connected to $c_{1}$ are $c, a^{\prime}, b_{1}, d_{1}$. Thus, $\sigma\left(\left\{a, c^{\prime}, b_{1}, d_{1}\right\}\right)=\left\{c, a^{\prime}, b_{1}, d_{1}\right\}$. Since $a \mapsto a^{\prime}, c^{\prime} \mapsto c$, and $b_{1} \not \mapsto b_{1}$, we have $b_{1}^{\prime}=d_{1}$ and we conclude that $G$ is as in Figure 14 .

Theorem 2 easily follows from Theorem 12 combined with Theorem 1 . Indeed, By Theorem 8 , any basic net $\Gamma$ on $\mathbb{R P}^{2}$ with more than one crossing is dual to a quadrangulation from $\overline{\mathcal{Q}}$. Hence $\Gamma$ can be obtained by successive special face splittings and vertex surroundings starting from a net dual to a $\overline{\mathcal{Q}}$-minimal quadrangulation of $\mathbb{R P}^{2}$. By Theorem $12, \overline{\mathcal{Q}}_{\text {min }}$ consists of $\check{g}_{2}^{5}$ and the quotients of those $\mathcal{Q}$-minimal quadrangulations of $S^{2}$ which admit a fix point free involution. By Theorem $1, \mathcal{Q}_{\min }=\left\{W_{n} \mid n \geq 3\right\}$ (double wheels). It is easy to check that $W_{n}$ admits a fix point free involution if and only if $n$ is odd. Thus, $\overline{\mathcal{Q}}_{\min }=\left\{\check{g}_{2}^{5}\right\} \cup\left\{\widetilde{W}_{n} \mid n\right.$ is odd, $n \geq 3\}$. It remains to note that $g_{2}^{5}$ is obtained from $g^{1}$ by a vertex surrounding and that the nets $\overline{(2 \times n)^{*}}$ are dual to $\widetilde{W}_{n}$.

\section{Computations}

Of course, the best way to generate basic nets in $\mathbb{R} \mathbb{P}^{2}$ is to write a program based on Theorem 2 and similar to plantri $[2,3]$ or, maybe, just to modify plantri. However, it takes too much efforts for somebody (like me) who is not familiar with plantri internal structure, so, I used a more lazy approach: I wrote a simple filter ppf for plantri (see [7]). It reads the output of plantri and selects only those planar graphs which admit an orientation reversing involution without fix points and fix edges. Since plantri called with -c2q option generates all simple quadrangulations of $S^{2}$, Theorem 8 ensures that we obtain in this way all simply embedded quadrangulations of $\mathbb{R P}^{2}$ (the dual graphs of basic nets with $\geq 3$ crossings) without repetitions and omissions.

This method is very slow, for example, we need to treat $5.45 \cdot 10^{13}$ simple quadrangulations of $S^{2}$ with 38 vertices to select only 1735808 simply embedded quadrangulations of $\mathbb{R P}^{2}$. Fortunately, plantri is so efficient that this can be done.

The program ppf can be used in pipe with plantri, for example:

$$
\text { plantri -c2q } 18 \text { | ppf }
$$

The output is almost the same as the plantri's ascii output but:

- The names of vertices are changed from $a, b, c, \ldots$ to $a, b, c, \ldots, A, B, C, \ldots$ so that the involution maps $a \mapsto A, b \mapsto B$, etc.

- We list the neighbourhoods of the lowercase vertices only.

For example, the first output line produced by the command (2) is

$$
9 \text { bcdef, aDg, agF, aFBH, aHI, aICD, bhic, DEg, gEF }
$$

which corresponds to the net $g_{1}^{8}$ in Figure 2. The corresponding net in $S^{2}$ is depicted in Figure 16 where $S^{2}$ is supposed to be glued out of the two disks so that the region names match each other. 


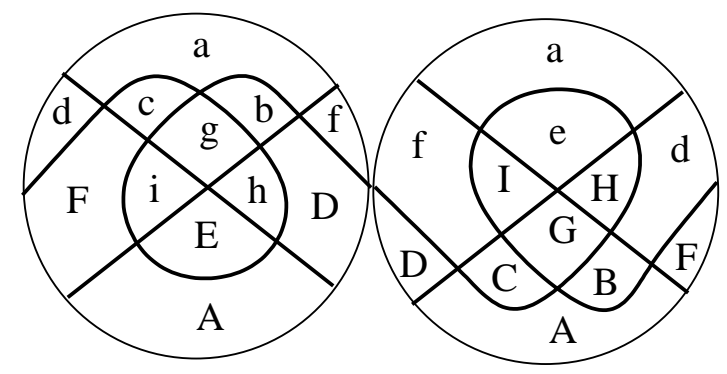

FIGURE 16

In Table $1, \bar{q}(\check{n})$ is the number of simply embedded quadrangulations of $\mathbb{R P}^{2}$ with $\check{n}=n+1$ vertices (the same as the number of basic nets on $\mathbb{R P}^{2}$ with $n$ vertices), $\bar{q}_{\text {bip }}(\check{n})$ is the number of those of them which are bipartite (the number of homologically trivial basic nets on $\mathbb{R P}^{2}$ with $n$ vertices), and $q_{2}(2 \check{n})$ is the number of simple quadrangulations of $S^{2}$ with $2 \check{n}$ vertices (the same as in [1; Table 2]), so, $q_{2}(2 \check{n})$ is the number of quadrangulations needed to be checked in our computation of $\bar{q}(\check{n})$.

\begin{tabular}{c|c|c|c|c}
$n$ & $\check{n}$ & $\bar{q}(\check{n})$ & $\bar{q}_{\text {bip }}(\check{n})$ & $q_{2}(2 \check{n})$ \\
\hline 3 & 4 & 1 & 0 & 1 \\
4 & 5 & 0 & 0 & 1 \\
5 & 6 & 2 & 1 & 3 \\
6 & 7 & 3 & 2 & 12 \\
7 & 8 & 6 & 3 & 64 \\
8 & 9 & 12 & 7 & 510 \\
9 & 10 & 37 & 22 & 5146 \\
10 & 11 & 95 & 57 & 58782 \\
11 & 12 & 293 & 174 & 716607 \\
12 & 13 & 923 & 554 & 9062402 \\
13 & 14 & 3086 & 1848 & 117498072 \\
14 & 15 & 10504 & 6291 & 1553048548 \\
15 & 16 & 36954 & 22052 & 20858998805 \\
16 & 17 & 131590 & 78361 & 284057538480 \\
17 & 18 & 475793 & 282420 & 3915683667721 \\
18 & 19 & 1735808 & 1027336 & 54565824458485 \\
\hline
\end{tabular}

TABLE 1 . Basic nets on $\mathbb{R P}^{2}$ with $n$ and on $S^{2}$ with $2 n$ vertices

\section{REFERENCES}

1. G. Brinkmann, S. Greenberg, C. Greenhill, B. D. McKay, R. Thomas, P. Wollan, Generation of simple quadrangulations of the sphere, Discrete Math. 305 (2005), 33-54.

2. G. Brinkmann, B. D. McKay, Fast generation of planar graphs, MATCH: Commun. Math.

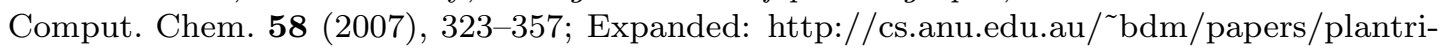
full.pdf.

3. G. Brinkmann, B. D. McKay, The program plantri, http://cs.anu.edu.au/^bdm/plantri. 
4. J. H. Conway, An enumeration of knots and links, and some of their algebraic properties, Computational Problems of Abstract Algebra (Proc. Conf., Oxford, 1967), Pergamon, Oxford, 1970, pp. 329-358; Available at http://www.math.ed.ac.uk/aar/knots/conway.pdf.

5. J. Drobotukhina, Classification of links in $\mathbb{R} P^{3}$ with at most six crossings, in "Topology of manifolds and varieties" (ed. O.Ya. Viro), Advances in Soviet Math., vol. 18, A.M.S., 1994, pp. $87-121$.

6. A. Nakamoto, Generating Quadrangulations of Surfaces with Minimum Degree at Least 3, Journal of Graph Theory 30 (1999), no. 3, 223-234.

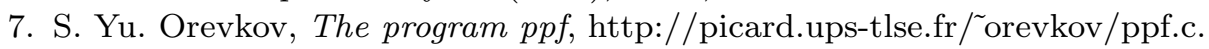

IMT, Université Paul Sabatier (Toulouse-3)

E-mail address: orevkov@math.ups-tlse.fr

Steklov Math. Institute, Moscow 